\title{
Mass Spectrometric Characterization of Peptides Associated with Molecules of the Major Histocompatibility Complex
}

\author{
Michael R. Pisano ${ }^{1}$ and Michael Ford ${ }^{2}$ \\ ${ }^{1}$ Cayman Chemical Company, Ann Arbor, MI, ${ }^{2}$ MS Bioworks LLC, Ann Arbor, MI
}

\section{Introduction}

Major histocompatibility complexes (MHCs or HLAs in humans) are expressed on the cell surface of all nucleated cells and act as the machinery for antigen presentation to $T$ cells in the acquired immune system. They function to display peptide fragments of processed self and foreign proteins (antigens) on the cell surface for inspection by $\mathrm{T}$ lymphocytes (CD8 ${ }^{+}$cytotoxic T lymphocytes (CTL) for MHC Class I, and CD4+ helper T lymphocytes for MHC Class II). Recognition of foreign antigens by $T$ cell receptors triggers an immediate $T$ cell activation and expansion, resulting in the destruction of the presenting cell by the CTL (Figure 1).

Characterizing the antigens involved in this process is paramount to understanding the immunogenicity of proteins and generating tools for targeted cell destruction (e.g., immunotherapy for targeting tumors). The molecular level characterization of peptides associated with MHC-I and -II requires a targeted protein complex enrichment by immunoprecipitation (IP), an unbiased peptide elution, and peptide analysis by mass spectrometry (MS). Using the human colorectal carcinoma cell line HCT116 as a case study, we present an optimized peptide enrichment methodology, stateof-the-art MS, and data processing for the sensitive and specific analysis MHC-I peptides.

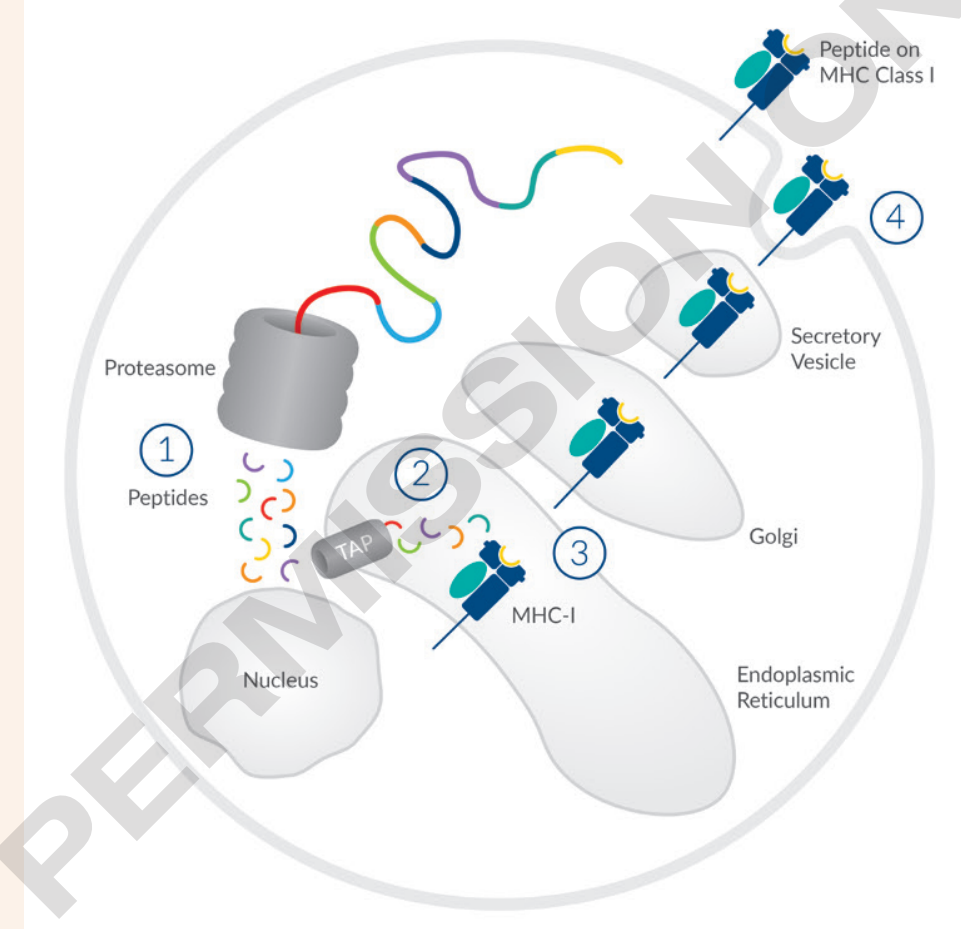

Figure $1 .^{3}$ Stages of MHC-I Peptide Presentation: 1) Protein processing in the proteasome; 2) peptide transport into the endoplasmic reticulum; 3) peptide assembly with Class I molecules; 4) peptide presentation on the cell surface.

\section{Materials and Methods}

HCT116 cells were grown to 80\% confluency, then further passaged 1:5 in HYPERFlasks and harvested by Accutase ${ }^{\circledR}$ treatment. After washing twice in PBS, harvested cell pellets with defined density were stored at $-80^{\circ} \mathrm{C}$. Cell pellets were lysed by homogenization at $4^{\circ} \mathrm{C}$ in $0.25 \%$ Deoxycholic Acid, 1\% Octylthioglucoside, $1 \mathrm{mM}$ EDTA, 0.2 mM lodoacetamide, and Roche HALT in PBS. The cleared lysate was applied to a washed immunoaffinity resin (Anti-human MHC-I Antibody (Clone W6/32) bound to Protein A-Sepharose ${ }^{\circledR} 4 \mathrm{~B}$ ) followed by overnight incubation at $4^{\circ} \mathrm{C}$, washes with $20 \mathrm{mM}$ Tris- $\mathrm{HCl}, 150 \mathrm{mM} \mathrm{NaCl}$, and elution of the MHC-I complex with multiple additions of $5 \%$ acetic acid. The eluent was passed through a 3 kDa MWCO spin filter.

Isolated peptides were desalted by StageTip and analyzed by nano LC-MS/MS with a Thermo Scientific Orbitrap Fusion ${ }^{\mathrm{TM}}$ Lumos $^{\mathrm{TM}}$ operating in Electron-Transfer/Higher-Energy Collision Dissociation (EThcD) mode. Peptides were loaded on a trapping column and eluted over a $75 \mu \mathrm{m} \times 50 \mathrm{~cm}$ analytical column at $300 \mathrm{~nL} / \mathrm{min}$ using a 2 hour reverse phase gradient; both columns were packed with PepMap RSLC C18, 2 um resin (Thermo Scientific). The mass spectrometer was operated in data-dependent mode, with the Orbitrap operating at 60,000 FWHM and 17,500 FWHM for MS and MS/MS, respectively. The instrument was run with a 3 second cycle for MS and MS/MS.

Data were processed with MaxQuant. ${ }^{1}$ MaxQuant employs the Andromeda search engine and data were searched against the UniProt Human reference proteome with no specific enzyme specificity, and N-terminal acetylation and methionine oxidation as variable modifications. The second peptide identification option in Andromeda was enabled. A false discovery rate of $1 \%$ was required for peptides; no protein false discovery rate was set. 


\section{Results}

Analysis of the MHC Class I peptidome by MS requires the selective enrichment of the MHC Class I complex since the MHC Class I molecules and peptides are present at low abundance. Once the complex is enriched, the MHC-associated peptides can be selectively isolated from the complex for further analysis. To address the sensitivity challenge, the selective immuno-enrichment was performed on a preparative scale. To achieve deep coverage of the Class I peptidome, we used $1 \times 10^{9}$ HCT116 cells as the input and 5 $\mathrm{mg} / \mathrm{mL}$ antibody load on the resin. This approach achieved peptide yields on the order of $5 \mu \mathrm{g}$ total peptide. The sensitivity obtained was also enabled by the application of EThcD. ${ }^{2}$

Analysis of the enriched peptides by LC-MS/MS results in approximately 2,000 unique 8-15-mer peptides per injection. Using features unique to the MaxQuant data processing platform, this increased to approximately 2,500 peptides per analysis. Figure 2 shows the comparisons of the HCT116 MHC Class I peptidomes obtained from the duplicate analysis of an enriched sample. The Pearson correlation coefficient of 0.98 obtained from the comparison of duplicate injections reflects the high degree of analytical reproducibility obtained with this optimized workflow.

Figure 3 is a histogram showing the peptide length against the number of observed peptides from the processed LC-MS/MS data. Our workflow shows greater than $60 \%$ enrichment of the 9-mer peptide population. The peptide sequence data from this 9-mer population can be used to confirm or predict HLA allele information by motif analysis.

The sensitivity we obtained was also enabled by the application of EThcD. EThcD is a fragmentation method that generates dual fragment ion series, enabling extensive peptide backbone fragmentation. We found this method of peptide fragmentation generated a 1.5-fold increase in the total peptide yield.

To learn more about MHC Peptide Sequencing Bioanalysis Services, please contact contractresearch@caymanchem.com orinfo@msbioworks.com.

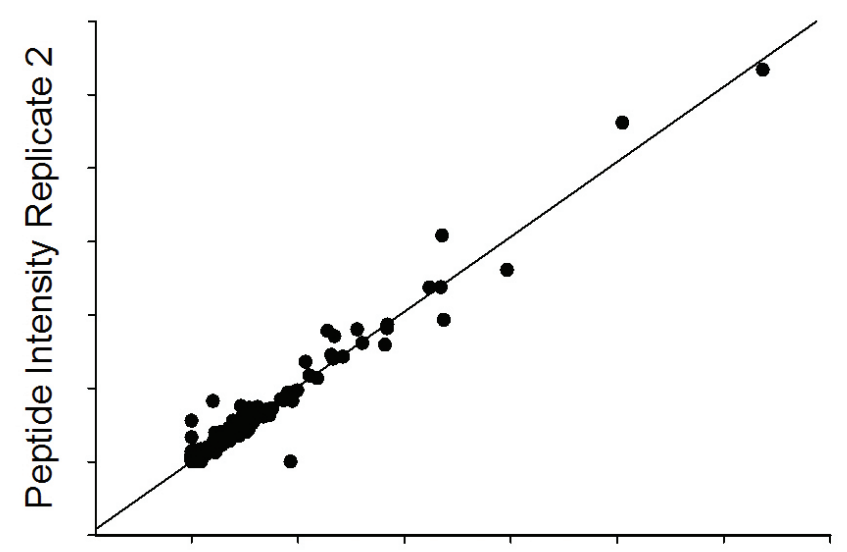

Peptide Intensity Replicate 1

Figure 2. Comparisons of the HCT116 MHC-I peptidomes obtained from the duplicate analysis of an enriched sample.

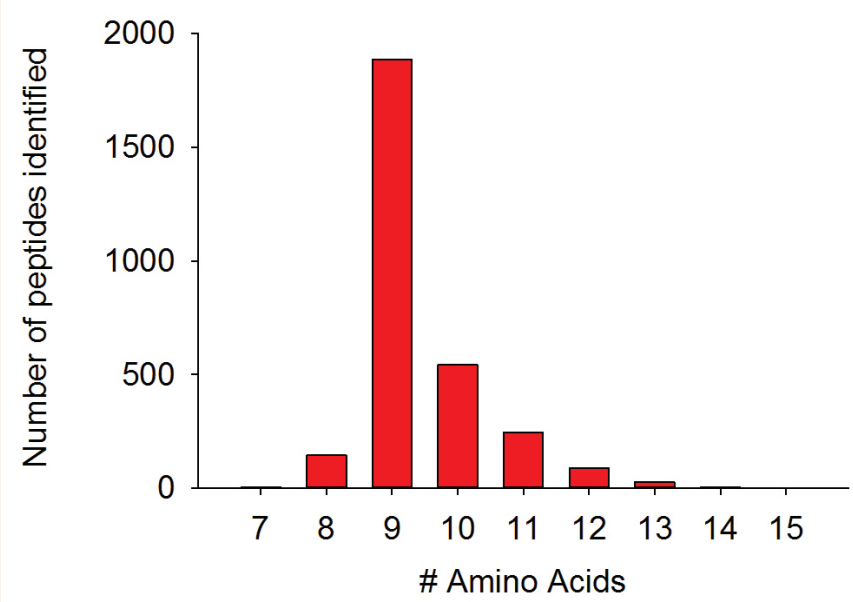

Figure 3. Histogram of the peptide length (\# Amino Acids) against the number of peptides from the processed LC-MS/MS data shows enrichment towards 9-mer peptides.

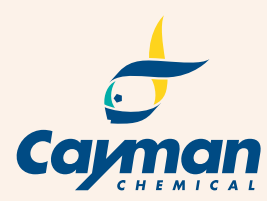

\section{References}

1. Cox, J. and Mann, M. Nat. Biotech. 26, 1367-1372 (2008).

2. Frese, C.K., Altelaar, A.F.M, van den Toorn, H., et al. Anal. Chem. 84(22), 9668-9673 (2012).

3. Image adapted from "MHC Class I processing" by Scray available under CC BY-SA 3.0.

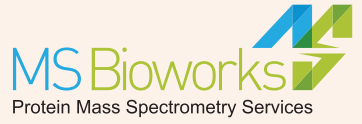

Protein Mass Spectrometry Services 